\title{
Hypnosis for PTSD: Evidence Based Placebo-Controlled Studies
}

\section{Barabasz $A^{*}$ and Barabasz $M$}

Washington State University, Pullman, Washington, USA

\begin{abstract}
A single manualized abreactive hypnosis session (5-6 hours) based on Ego State Theory (EST) was recently subjected to two placebo-controlled investigations meeting evidence-based criteria. Thirty-six patients in study \#1 and 30 patients in study \#2 who met PTSD criteria were exposed to either 5-6 hours of a manualized treatment or a placebo in a single session. Abreactive hypnosis emphasized hypnotically activated "reliving" of the trauma experience to physical and psychological exhaustion. In study \#1 hypnosis and control group's reduced PTSD checklist (PCL) scores immediately post treatment (placebo PCL score mean reduction 17. 34 and EST treatment PCL mean reduction 53.11). However, only the hypnosis patients maintained significant treatment effects at followups. Study \#2 used the Davidson Trauma Scale (DTS), Beck Depression II (BDI - II), and Beck Anxiety Scales (BAI). Only the hypnosis group showed significant positive effects from pretreatment to all post treatment measurement periods. Abreactive EST was shown to be a highly effective and durable treatment for PTSD. Apparently, EST works because it is emotion focused, activates sub-cortical structures, and because the supportive, interpretive therapist reconstructs the patient's personality to be resilient and adaptive.
\end{abstract}

Keywords: PTSD; Ego state therapy; Emotion focused therapy; Hypnosis

\section{Introduction}

Abreactive hypnosis, the key component of Ego State Therapy (EST), evolved from a psychodynamic understanding of personality as a product of an individual's ego states [1-6]. Exposure to a traumatic event activates subcortical structures. Thus, trauma memories become encoded in the subcortical-subconscious brain regions. Watkins [7] foreshadowed a bottom-up explanation of the neurobiological underpinnings of trauma reactions in his classic book Hypnotherapy of the War Neuroses. Decades later, van der Kolk [8] explained that traumatic memories stay "stuck" in the brain in the nonverbal, subconscious, subcortical region (hippocampus) where they are not accessible to the frontal lobes-the understanding, thinking, and reasoning part of the brain.

As explained in detail elsewhere [4], sensory input triggering a trauma flashback stimulates hormonal secretions and influences the activation of brain regions involved in attention and memory. This discovery sheds light on why conscious control over the patient's actions is limited [8]. Reminders of the past can activate specific neurobiological responses. Thus survivors of trauma are vulnerable to react with irrational and sub-cortically initiated responses that are irrelevant, and even harmful, in present circumstances or situations [8]. Triggered responses are often found to be coupled to ego state dependent defense mechanisms learned in early life $[9,10]$. A stimulus associated with the trauma experienced in the past becomes present [8] precluding patients from sufficiently integrating trauma memories into conscious mental frameworks. This makes them inaccessible by the top-down (talk about the trauma/reframe) cognitive-processing therapies which focus on only conscious processes in an attempt to impact emotional responses which, at best, lead only to superficial functioning. The patient remains vulnerable to flashbacks and retraumatization [11-17].

Trauma-imagery symptom-provocation paradigms used in neuroimaging studies [18-21] have shed light on the physiology of the bottom-up conceptualization of accessing brain areas where trauma is encoded. Consistent findings revealed neurobiological shifts that were specifically associated with the focused attention and heightened arousal of reliving the trauma. The findings showed that patients who were exposed to traumatic reminders (triggers) displayed increased cerebral blood flow in the right medial orbito-frontalcortex, insula, amygdala, and anterior temporal pole. However, in the left anterior prefrontal cortex coincident relative deactivation was noted. The deactivation was especially dramatic in Broca's area. This expressive speech center of the brain is essential to communicate thoughts and feelings. Subsequent research showed that when a previous personal trauma is triggered, regions of the brain that experience intense emotions are activated. The activity of brain structures involved in the control of emotions and the translation of experience into communicable language is simultaneously decreased [18-21]. PTSD patients may display inappropriate emotions, bizarre reactions, and/or "freeze up" when confronted with seemingly normal challenges [11]. The Broca's area of the brain may shut down, so these patients become extremely limited in their ability to communicate what it is they are feeling. In an attempt to cope with these events, those with PTSD are thought to regress to primary process thinking $[9,10]$. Thus, they respond to reminders of the past by automatically engaging in thinking and actions that were once appropriate and adaptive at a primary level but are now maladaptive in response to the current presenting stimuli. These findings are consistent with Jackson's explanation [22] that conceptualized the brain as hierarchically organized from the bottom up.

The imprint of the trauma is not accessible via the verbal, intellectual, defensive, or executive parts of the brain because it

*Corresponding author: Prof. Arreed Barabasz, Ph.D., ABPP, Editor, International Journal of Clinical and Experimental Hypnosis, Washington State University, Cleveland Hall, P.O. BOX 642136, Pullman, WA 99161-2136, USA, Tel: (509) 3358166; E-mail: arreed_barabasz@wsu.edu

Received October 04, 2013; Accepted November 26, 2013; Published November 28, 2013

Citation: Barabasz A, Barabasz M (2013) Hypnosis for PTSD: Evidence Based Placebo-Controlled Studies. J Trauma Treat S4: 006. doi:10.4172/2167-1222.S4006

Copyright: (c) 2013 Barabasz A, et al. This is an open-access article distributed under the terms of the Creative Commons Attribution License, which permits unrestricted use, distribution, and reproduction in any medium, provided the original author and source are credited. 
is deeply entrenched in the subcortical subconscious (amygdala, hippocampus), which are at best only peripherally affected by thinking and cognition $[1,23]$. Abreactive hypnosis focuses on changing the way people regulate these core functions. Clearly, therapy should not be limited to talking about trauma or cognitive reframing at the purely conscious levels but instead activate the primary-process regions to an intense reliving of the actual events [24]. The purpose of our research was to determine whether or not abreactive hypnosis, applied within an Ego State Theory conceptualization, is an effective treatment for PTSD.

\section{The 5-6 Hour Single Session Procedure}

The procedure consists of five distinct phases which are described in detail in Barabasz et al. [24] and fully elaborated, with specific hypnotic scripts and detailed protocols, in our treatment manual ${ }^{\star}$ [24]. The German version, Barabasz et al. [25] is culturally adapted to commonly used German clinical phraseology. The Spanish Edition [26] is specifically written for Mexico and fluent Spanish speaking Mexican-Americans.

The elements of the 5-phase procedure are, 1) qualifying the patient (the trauma must be accessible, circumscribed, and orienting) to help ensure likely benefit from the single-session approach and provide a rapid orientation to EST. It includes administration of the Clinician Administered PTSD Scale (CAPS) [27], 2) contacting ego states which includes debunking myths about hypnosis and introducing the patient to hypnosis via a hypnotic-like experience, (for example, Chevreul's pendulum swing test [28]). Then the manualized hypnotic induction identifies the ego state(s) harboring the trauma, 3) diagnostic exploration of each known ego state, 4) ego state mapping, and 5) specific protocols for resolution of internal conflicts via hypnotically induced abreactions.

Abreactive hypnosisis the key component of the single session approach, but all 5 phases are essential. The protocols and scripts are involved attention to the past, present, and future. Efficiency of the single 5-6 hour session is achieved because of the abreactive intensity and repetition. A single abreaction, no matter how intense is an insufficient basis for lasting adaptive change. Abreactions are repeated 3 or 4 times within the single 5-6 hour EST session. When carried out properly, each subsequent abreaction is experienced less violently until complete resolution has been reached.

\section{Evidence based Abreactive Hypnosis: Two Placebo Controlled Studies}

As is evidenced by five world congresses, EST is viewed as the therapy of choice for a range of trauma based disorders. Earlier this year the 5th World Congress on Ego State Therapy was held in Johannesburg, South Africa. The 4th World Ego State Congress held in Heidelberg, Germany in November 2011 drew over 1500 attendees and the International Hypnosis Congress with over 2400 attendees, held Bremen, Germany in October 2012 devoted an entire plenary session day to EST. Yet, despite its international acceptance as a common treatment of choice, EST has been largely ignored in the United States. The Department of Defense,

Veterans Administration, and the American Psychological Association only recognize, as evidence based, Cognitive Processing [29] and EMDR [30].

Until 2013, EST lacked placebo-controlled research meeting evidence-based criteria. To meet this need two independently conducted studies were carried out $[5,6]$. Both provide strong evidence that a single 5-6 hour manualized abreactive EST session is an effective and durable treatment for PTSD. Summaries of the studies appear below as well as the addition of12-month follow-up data.

Barabasz et al. [5] and Christensen et al. [6] used similar research designs but different dependent measures. All patients $(n=36)$ Barabasz et al. [5] and $(n=30)$ Christensen et al. [6] met DSM-IV TR and Clinician Administered PTSD Scale (CAPS) [31] criteria for PTSD. All 36 in the Barabasz study scored above average (scores 3-5 on a 5 point scale) on the Stanford Hypnotic Clinical Scale (SHCS) [32]. The Hypnotic Induction Profile (HIP) [33] indicated that all in the Christensen study were also responsive to hypnosis (scores $4-10$ on the 10 point scale). The dependent measure for the Barabasz study was the PCL [27] which was administered in the civilian or military version as appropriate to the patient. Placebo Control group Ss were exposed to information about PTSD from the United States Veterans Administration online "About Face" app (http://www.ptsd.va.gov/public/about_face.html). By clicking on a photograph, from the dozens provided. Each Ss, in the presence of the empathic experimenter/therapist is exposed to information about PTSD. This included "How I knew I had PTSD. How PTSD affects the people you love," "Why I didn't ask for help right away," When I knew I needed help," My advice to you," etc. The Christensen study used the DTS [3], the BAI [34] and the BDI-II [35]. The fifteen Ss in the active control were exposed to a Veteran's Administration approved video treatment which provided them with general information about PTSD and the Counting Method (Ochberg). The Counting Method is a technique designed for "mastering traumatic memories" aiming to desensitize traumatized subjects (Johnson and Lubin). It is designed to help Ss process their traumatic memories without evoking intense affect. Both studies assigned patients in balanced order. Both used the same treatment manual for the single 5-6 hour co-therapy session. The Barabasz study collected PCL data pre and post session. Follow up PCL data was collected at 4 weeks, 16 to 18 weeks and 12 months. All 36 volunteers completed the treatment or control condition.

\section{Results}

To examine the effects of the EST condition and time, a repeated measures analysis of variance (RANOVA) was performed with treatment level as the between-subjects factor and time as the withinsubjects factors. Effect sizes ( $\eta 2, d)$ [36] were reported to assist with interpretation of the statistical test results. Table 1 contains the descriptive information by group across time.

Standard data screening and assumptions checking were conducted before the analysis of the outcome data. Skewness and kurtosis were assessed for the dependent variable across time. No scores in these conditions were found to be problematic, Skew $=-2.20$ to 1.50 , Kurtosis range $=-0.82$ to 5.60 . Levene's test for homogeneity of variance was significant at the posttest and 4 week follow-up, $\mathrm{p}<0.01$ but not for the pretest and 16-18 week follow-up results. This was viewed as a reflection of the treatment reducing the variability in scores during this time as the variability is the same as control participants at pretest and 16-18 weeks post treatment. The assumption of homogeneity of covariance matrices, tested via Box's $M$, was significant, $p<0.01$. The assumption of sphericity was met, $\mathrm{p}>0.05$. Given the sample size, the resulting $\mathrm{F}$ values and the associated power, there was not concern over minor violations of assumptions. Multivariate normality could be assumed with the proportion of Mahalanob is Distances not deviating sufficiently from the $50^{\text {th }}$ percentile of the chi-square distribution.

There was a significant effect of group, $F(1,34)=84.73, p<0.01$, 


\begin{tabular}{|c|c|c|c|c|c|}
\hline \multicolumn{7}{|c|}{ PCL Means } \\
\hline & Pretest & Posttest & 4 weeks & $16-18$ wks & 12 months \\
\hline Control & 77.00 & 59.66 & 68.66 & 72.27 & 77.40 \\
\hline Treatment & 77.11 & 24.00 & 30.66 & 33.05 & 28.47 \\
\hline
\end{tabular}

Table 1: PCL Means for Control and Experimental Groups.

partial $\eta 2=0.714$. Across time the control group had a significantly higher mean, $\mathrm{M}=69.40$, compared to the experimental group, $\mathrm{M}=41.21$. There also was a significant effect of time, $F(3,102)=85.75, p<0.01$, partial $\eta 2=0.716$, and time by group interaction, $F(3,102)=33.24$, $\mathrm{p}<0.01$, partial $\mathrm{n} 2=0.494$.

As seen in Table 1, the experimental group appeared to have lower mean scores at each time point with the exception of the pretest assessment. All differences between groups were statistically significant except for the pretest difference. The effect size differences at posttest, $\mathrm{d}=2.91$, at 4 weeks, $\mathrm{d}=4.87$, and at $16-18$ weeks follow-up, $\mathrm{d}=2.45$, revealed large differences. The largest difference was near 5 standard deviations at 4 weeks follow-up. The difference was greater than 2 standard deviations at the 16 to 18 weeks follow-up point.

Figure 1 displays the average scores by group across time. As can be seen in Figure 1 both the treatment and control groups did not differ on the pretest assessment. After this point in time, the treatment group has lower scores on the dependent variable across time compared to the control group. Simple effect analysis was used to dissect the interaction revealed that the control group had a significant difference from pretest to posttest $(\mathrm{p}<.01)$, yet this significant difference was not maintained over time as the remaining two differences compared to pretest were not significant, $p>0.05$. In fact, there was a significant increase between posttest and the 16-18 week follow-up suggesting a trend of returning to beginning status. In contrast, the treatment group had a significant decrease between pretest and each post assessment, $\mathrm{p}<0.01$. There were no statistically significant declines in scores after the posttest assessment, yet there were no statistical increases in scores, as was seen in the control condition.

The Christensen study collected DTS, BAI and BDI-II data pre and post the treatment and control sessions as well as at 4 weeks and at 12 weeks. Two-way analyses of variance (ANOVAs) (2 groups X 4 measurement periods) were calculated on the DTS, BDI-II) and BAI scores. Follow-up univariate ANOVA's and Tukey's were calculated.
Because of the distributions of the data, non-parametric tests were calculated (Mann-Whitney U and Wilcoxon Signed-Ranks tests). One way ANOVA's computed for the DTS scores, BDI-II scores and BAI scores at pre-test revealed no significant differences between the EST and the active control group at pre-test for any measure (DTS, $\mathrm{F}(1,28)=.17, \mathrm{p}>.68$; BDI-II, $\mathrm{F}(1,28)=1.88, \mathrm{p}>.18$; BAI, $\mathrm{F}(1,28)=.03$, $\mathrm{p}>$.87).

DTS was conceptualized to be the measure most directly relevant to the primary purpose of the study. The $2 \times 4$ analysis of variance revealed a significant within-subjects effect $(\mathrm{F}(3,26)=44.48, \mathrm{p}<.001$, Wilks' Lambda=.1639), a significant between groups effect $(F(3,26)=8.24$, $\mathrm{p}<.0005$, Wilks' Lambda $=.5126)$ and a significant interaction effect (trial X group) $(\mathrm{F}(3,84)=5.44, \mathrm{p}<.007)$. Follow-up univariate ANOVAs revealed significant decreases in PTSD symptoms for the EST group from pre-treatment to post-test $(\mathrm{F}(1,28)=15.46, \mathrm{p}<.0005$ and one month follow-up $(\mathrm{F}(1,28)=7.68, \mathrm{p}<.009)$.

Examination of the data revealed a greater than $34 \%$ increase in variance at the three month follow-up (compared to post-treatment and one month data) which can be typical of small $\mathrm{N}$ therapeutic intervention studies. The distribution of scores became non-normal at three months, which violates the normalcy assumption required for parametric analyses. Therefore, a non-parametric approach was adopted to analyze the three months data. A Wilcoxon Signed-Ranks test was calculated. The result $(\mathrm{Ns}-\mathrm{R}=15, \mathrm{~T}=0, \mathrm{p}<.001)$ shows $\mathrm{EST}$ continued to have a significant positive effect on DTS scores at the three-month follow-up.

Tukeys Studentized HSDs were computed to compare the EST and the active control group. The results indicated significantly lower DTS scores for EST versus the active control condition at post-treatment (Studentized critical value $=2.89$, minimum significant difference 22.09, Control mean 78.2, EST mean 35.8, p<.05) and at one-month followup (Studentized critical range $=2.89$, minimum significant difference 22.95, Control mean=60.20, EST mean $=29.13, \mathrm{p}<.05$ ). Due to the non-normal distribution of scores at the three-month follow-up, the non-parametric Mann Whitney $U$ was calculated. The result yielded a $\mathrm{Z}=2.31, \mathrm{p}<.05$ showing EST produced significantly lower DTS scores at three months than the active control condition.

The overall ANOVA of the BDI-II revealed a significant within

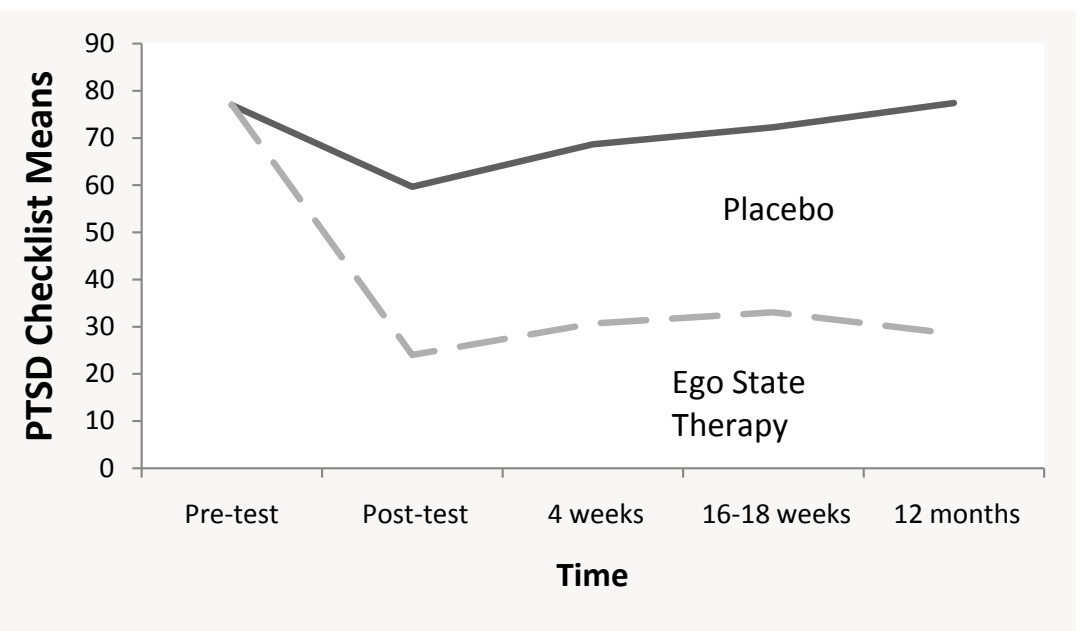

Figure 1: Ego State and Control Group PTSD Checklist Results over 12 months 
groups result $(\mathrm{F}=(3,26), 38.19, \mathrm{p}<.001$, Wilks' Lambda $=.18)$ and a significant between groups result $(\mathrm{F}=(3,26), 20.64, \mathrm{p}<.0001$, Wilks' Lambda $=.29$ ). WIlcoxon Signed Ranks test results showed significantly lower BDI-II scores at post-test (Ns-R=15, T=6.2, $\mathrm{p}<.001$ ), at one month (Ns-R=15, $\mathrm{T}=1.5, \mathrm{p}<.001$ ), and at three months (Ns-R=15, $\mathrm{T}=7$, $\mathrm{p}<.001)$.

Tukeys Studentized HSD results indicated significant effects for EST versus the active control condition at post-treatment (Studentized critical value $=2.90$, minimum significant difference 7.90 , Control mean 21.73, EST mean 5.80, $\mathrm{p}<.05$ ). Given that the score distributions were not normally distributed at one month and three months, MannWhitney U tests were calculated. The results showed EST produced significantly lower BDI-II scores versus the active control condition at one month $(\mathrm{Z}=3.77, \mathrm{p}<.01)$ and at three months $(\mathrm{Z}=2.42, \mathrm{p}<.05)$.

The overall ANOVA of the BAI revealed a significant within groups result $(\mathrm{F}(3,26)=21.46, \mathrm{p}<.0001$, Wilks' Lambda $=.28)$ and a significant between groups result $(\mathrm{F}(3,26)=7.13, \mathrm{p}<.001$, Wilks' Lambda $=.54)$. Follow-up ANOVA results $\mathrm{F}(1,28)=15.4, \mathrm{R} 2=.35, \mathrm{p}<.0005$ showed EST produced significantly lower BAI scores at post-test versus pretreatment. Wilcoxon Signed Ranks test results showed EST produced significantly lower scores than pre-test at one month (Ns-R=15, T= -3 , $\mathrm{p}<.001$ ) and at three months (Ns- $\mathrm{R}=15, \mathrm{~T}=2, \mathrm{p}<.001$ ).

Tukeys Studentized HSD results indicated significant effects for EST versus the active control condition at post-treatment (Studentized critical value $=2.90$, minimum significant difference 7.13 , Control mean 20.6, EST mean 6.93, $\mathrm{p}<.05$ ). Due to the non normal distribution of scores at one and three months follow-up, Mann Whitney U tests were calculated which showed EST produced significantly lower BAI scores than the active control condition at one month $(\mathrm{Z}=2.44, \mathrm{p}<.05)$ and at three months $(\mathrm{Z}=4.9, \mathrm{p}<.01)$.

\section{Discussion}

The major findings of the two studies show that a single 5-6 hour session of manualized abreactive EST is an effective treatment at immediate post -treatment and over repeated follow-ups using the most commonly accepted measures of PTSD symptoms. The placebo groups appeared to show a significant immediate post treatment effects indicating that the placebo conditions functioned as believable treatment conditions. Yet, for example, the apparent placebo effects in the Barabasz study (a reduction in PCL scores of 17.34 points for controls) are clinically meaningless when contrasted with the posttreatment effects produced by the abreactive hypnosis ego state group (a reduction in PCL scores of 53.11 points). The immediate posttreatment significant placebo effects vanished at the first and subsequent follow-ups while the clinically meaningful ego state treatment effects were maintained over time in both studies.

Beyond the quantitative data, the expressions of relief from years of symptoms by the abreactive hypnosis EST treated patients were often profound in both the nature of the verbalizations and the appearance of relief expressed in relaxed posture and pleasant facial expression. Instead of a repressed pain the trauma event became a simple memory, resilient to re-traumatization.

\section{Footnote}

*The spiral-bound manual (clinical edition; Barabasz, Barabasz, Christensen, et al., 2012) is available only to licensed health care professionals and qualifies researchers at nominal cost by emailing the first author (arreed barabasz@wsu. edu). The authors ask that this copyrighted material not be made available to the public as it could be misused.

\section{References}

1. Barabasz A, Barabasz M, Watkins JG (2011) Single session manualized ego state therapy (EST) for combat stress injury, PTSD, and ASD, Part 1: The theory. International Journal of Clinical and Experimental Hypnosis 59: 379391.

2. Weathers FW, Keane TM, Davidson JR (2001) Clinician-administered PTSD scale: a review of the first ten years of research. Depress Anxiety 13: 132-156.

3. Davidson JR, Book SW, Colket JT, Tupler LA, Roth S, et al. (1997) Assessment of a new self-rating scale for post-traumatic stress disorder. Psychol Med 27 : 153-160.

4. Barabasz A (2013) Evidence based abreactive ego state therapy for PTSD. American Journal of Clinical Hypnosis 56: 54-65.

5. Barabasz A, Barabasz M, Christensen C, French B, Watkins JG (2013) Efficacy of single-session abreactive ego state therapy for combat stress injury, PTSD, and ASD. Int J Clin Exp Hypn 61: 1-19.

6. Christensen C, Barabasz A, Barabasz M (2013) Efficacy of Abreactive Ego State Therapy for PTSD. International Journal of Clinical and Experimental Hypnosis 61: 20-37.

7. Watkins JG (1949) Hypnotherapy of war neuroses. New York: Ronald.

8. van der Kolk B (1994) Psychobiology of posttraumatic stress disorder. In Panksepp, J. (Ed.), Textbook of biological psychiatry, (pp. 319-344). New York: Wiley-Liss.

9. Barabasz A, Christensen C (2006) Age regression: tailored versus scripted inductions. Am J Clin Hypn 48: 251-261.

10. Christensen C, Barabasz A, Barabasz M (2009) Effects of an affect bridge for age regression. Int J Clin Exp Hypn 57: 402-418.

11. Bhuvaneswar C, Spiegel D (2013) An eye for an I: a 35-year-old woman with fluctuating oculomotor deficits and dissociative identity disorder. Int J Clin Exp Hypn 61: 351-370.

12. Cunningham PF, Ramos $P$ (2012) Involuntary experiencing and the performance of hypnotic test suggestions. Int J Clin Exp Hypn 60: 416-431.

13. Spiegel D (1984) Multiple personality as a post-traumatic stress disorder. Psychiatr Clin North Am 7: 101-110.

14. Spiegel D (1991) Neurophysiological correlates of hypnosis and dissociation. J Neuropsychiatry Clin Neurosci 3: 440-445.

15. Spiegel D (1998) Hypnosis and implicit memory: automatic processing of explicit content. Am J Clin Hypn 40: 231-240.

16. Spiegel D (2003) Negative and positive visual hypnotic hallucinations: attending inside and out. Int J Clin Exp Hypn 51: 130-146.

17. Spiegel D, King R (1992) Hypnotizability and CSF HVA levels among psychiatric patients. Biol Psychiatry 31: 95-98.

18. Hull AM (2002) Neuroimaging findings in post-traumatic stress disorder Systematic review. Br J Psychiatry 181: 102-110.

19. Lanius RA, Williamson PC, Densmore M, Boksman K, Gupta MA, et al. (2001) Neural correlates of traumatic memories in posttraumatic stress disorder: a functional MRI investigation. Am J Psychiatry 158: 1920-1922.

20. Lindauer RJ, Booji J, Habraken JBA, Uylings HB, Olff M, et al. (2004) Cerebra blood flow changes during script driven imagery in police officers with posttraumatic stress disorder. Biological Psychiatry 56: 853-861.

21. Van der Kolk B (2007) New frontiers in trauma treatment. Presented at the Institute for the Advancement of Human Behavior, Seattle, WA.

22. Jackson $\mathrm{J}$ (1958) Evolution and dissociation of the nervous system. In J.J. Taylor (Ed.), Selected Writings of John Hughlings, (pp. 45-118). London, UK: Stapes Press.

23. Watkins JG, Barabasz A (2008) Advanced Hypnotherapy: Hypnodynamic Techniques. New York, London: Routledge Taylor \& Francis.

24. Barabasz A, Barabasz M, Watkins JG (2012) Single Session Manualized Ego State Therapy (EST) for Combat Stress Injury, PTSD, and ASD: The procedure. International Journal of Clinical and Experimental Hypnosis 60: 370-381.

25. Barabasz A, Barabasz M, Christensen C, Riegel S, Watkins JG (2013) Abreagierende ego state therapie ein wegeiser für kamphbding te störungen, 
Citation: Barabasz A, Barabasz M (2013) Hypnosis for PTSD: Evidence Based Placebo-Controlled Studies. J Trauma Treat S4: 006. doi:10.4172/21671222.S4-006

Page 5 of 5

PTBS und ASD. Self-published manuscript, Arreed Barabasz, Palouse, Washington.

26. Barabasz A, Barabasz M, Christensen C, Sanchez-Armass O, Watkins J (2014In final revision). Abreactive Ego State Therapy, Manual for Combat Stress Injury, PTSD \& ASD (Mexican Edition in Spanish). Self-published manuscript, Arreed Barabasz, Palouse, Washington.

27. Bliese PD, Wright KM, Adler AB, Cabrera O, Castro CA, et al. (2008) Validating the primary care posttraumatic stress disorder screen and the posttraumatic stress disorder checklist with soldiers returning from combat. J Consult Clin Psychol 76: 272-281.

28. Barabasz A, Watkins JG (2005) The Hypnotherapeutic Techniques, 2E. New York: Brunner-Rutledge.

29. Foa E, Hembree E, Rothbaum B (2007) Prolonged exposure therapy for PTSD: Emotional processing of traumatic experiences, therapist guide. Oxford, United Kingdom: Oxford University Press.

30. Shapiro $F$ (2002) EMDR 12 years after its introduction: past and future research $\mathrm{J}$ Clin Psychol 58: 1-22.
31. Blake DD, Weathers FW, Nagy LM, Kaloupek DG, Gusman FD, et al. (1995) The development of a Clinician-Administered PTSD Scale. J Trauma Stress 8: 75-90.

32. Morgan A, Hilgard JR (1975) The Stanford Hypnotic Clinical Scale for adults. In E. Hilgard \& J Hilgard (Eds.), Hypnosis in the relief of pain (pp 134-147). Los Altos, CA: Kaufmann.

33. Spiegel H, Spiegel D (2004) Trance \& treatment: Clinical uses of hypnosis Washington, DC: American Psychiatric.

34. Beck AT, Steer RA (1993) Manual for the Beck Anxiety Inventory. San Antonio TX: Psychological Corporation.

35. Beck AT, Steer RA, Brown GK (1996) Manual for the Beck Depression Inventory-II. San Antonio, TX: Psychological Corporation.

36. Cohen J (1988) Statistical power analysis for the behavioral sciences. Hillsdale $\mathrm{NJ}$ : Erlbaum.
This article was originally published in a special issue, Post Traumatic Stress Disorders handled by Editor(s). Dr. Agius M, University of Cambridge, UK 